\title{
Expanding the use of monoclonal antibody therapy of cancer by using ionising radiation to upregulate antibody targets
}

\author{
M M Wattenberg ${ }^{1}$, A R Kwilas ${ }^{1}$, S R Gameiro ${ }^{1}$, A P Dicker ${ }^{2}$ and J W Hodge ${ }^{\star, 1}$ \\ ${ }^{1}$ Laboratory of Tumor Immunology and Biology, Center for Cancer Research, National Cancer Institute, National Institutes \\ of Health, 10 Center Drive, Room 8B13, Bethesda, MD 20892, USA and 2 Department of Radiation Oncology, Thomas Jefferson \\ University, 111 S. 11th Street, Philadelphia, PA 19107, USA
}

Background: Monoclonal antibody (mAb) therapy for the treatment of solid and haematologic malignancies has shown poor response rates as a monotherapy. Furthermore, its use is limited to tumours expressing certain molecular targets. It has been shown that single-dose radiation can induce immunogenic modulation that is characterised by cell-surface phenotypic changes leading to augmented tumour cell/cytotoxic T-cell interaction.

Methods: We examined radiation's ability to upregulate mAb therapy targets. We also used radiation to sensitise tumour cells to antibody-dependent cell-mediated cytotoxicity (ADCC).

Results: Radiation significantly increased cell-surface and total protein expression of mAb targets HER2, EGFR, and CD20. Focusing on HER2, targeted by trastuzumab, we observed significant upregulation of HER2 following radiation of 3 out of 3 breast cancer cell lines, one of which was triple negative, as well as in residential stem-cell populations. HER2 upregulation was sustained up to $96 \mathrm{~h}$ following radiation exposure and was largely dependent on intracellular reactive oxygen species. Improved ADCC and sensitisation to the antiproliferative effects of trastuzumab demonstrated the functional significance of radiation-induced HER2 upregulation.

Conclusions: We show that single-dose radiation enhances mAb therapy. These findings highlight a mechanism for combining radiation with immunotherapy and expand the patient population that can be treated with targeted therapy.

Ionising radiation is commonly used for the treatment and palliation of cancer. The goal of radiation therapy is to directly kill tumour cells by inducing DNA damage (Kwilas et al, 2012). Alternative methods of tumour control that exploit the immunestimulating potential of radiation therapy are currently being explored (Kwilas et al, 2012; Formenti and Demaria, 2013). We have previously described radiation-induced immunogenic modulation of tumour cells (Chakraborty et al, 2003, 2004; Garnett et al, 2004; Reits et al, 2006). Tumour cells exposed to sublethal radiation undergo phenotypic changes, including upregulation of MHC class I, tumour-associated antigens (TAAs), adhesion molecules, and death receptors. This immunogenic modulation translates into improved interaction between $\mathrm{CD}^{+}$cytotoxic $\mathrm{T}$ cells and the upregulated molecules, leading to enhanced immunemediated tumour cell killing. We hypothesised that single-dose radiation could alter the surface phenotype of tumour cells and enhance monoclonal antibody $(\mathrm{mAb})$ therapy by upregulating the target molecule.

We chose United States Food and Drug Administration (FDA)approved $\mathrm{mAb}$ targets that are expressed on tumours for which the standard of care includes radiation. HER2, a receptor tyrosine kinase, is overexpressed in 15-23\% of breast cancers (Mohd Sharial et al, 2012); EGFR, a related receptor tyrosine kinase, is overexpressed in $90 \%$ of head and neck squamous cell carcinomas (HNSCCs); and CD20 is expressed by B-cell lymphomas (Dotan et al, 2010; Tejani et al, 2010). The FDA has approved an $\mathrm{mAb}$

\section{*Correspondence: Dr JW Hodge; E-mail: jh241d@nih.gov}

Received 15 November 2013; revised 17 January 2014; accepted 20 January 2014; published online 20 February 2014 
therapy for each of these three molecules: trastuzumab for HER2, cetuximab for EGFR, and rituximab for CD20 (Scott et al, 2012). Although these $\mathrm{mAb}$ have shown clinical benefit in the form of improved overall survival, issues of resistance and poor response rates have limited their efficacy (Friedberg, 2011; Markovic and Chung, 2012; Mohd Sharial et al, 2012). We hypothesised that if radiation could increase expression of the $\mathrm{mAb}$ targets such as HER2, EGFR, and CD20, a greater number of cancer patients could benefit from $\mathrm{mAb}$ therapy.

Monoclonal antibody functions by, among other mechanisms, inhibiting cell proliferation and engaging the immune system through antibody-dependent cell-mediated cytotoxicity (ADCC) (Scott et al, 2012; Tagliabue et al, 2012). Antibody-dependent cellmediated cytotoxicity is common to several mAb of the IgG1 isotype, including trastuzumab, cetuximab, and rituximab (Scott et al, 2012). We focused on the potential for radiation to improve the functional outcome of mAb therapy as measured by ADCC and inhibition of proliferation.

This is the first study to report that (a) radiation exposure significantly increased cell surface and total protein expression of multiple targets of mAb therapy, including HER2, EGFR, and CD20; (b) radiation upregulated HER2 in 3 out of 3 breast cancer cell lines, one of which was triple negative; (c) radiation significantly upregulated HER2 in a residential stem-cell population; (d) HER2 upregulation after radiation was largely dependent on intracellular production of reactive oxygen species (ROS); and (e) increased expression of HER2 after radiation improved trastuzumab-mediated ADCC and sensitised breast cancer cells to the antiproliferative effects of trastuzumab.

Our finding that single-dose radiation enhances mAb therapy highlights a mechanism for using radiation in combination with immunotherapy and expands the patient population that can be treated with targeted therapy.

\section{MATERIALS AND METHODS}

Cell culture. Human breast carcinoma cells (MCF-7, ZR75-1, and MDA-MB-231) and non-Hodgkin's B-cell lymphoma (NHL) cells (Ramos) were obtained from American Type Culture Collection (Manassas, VA, USA) and cultured in a medium designated by the provider for propagation and maintenance. Human HNSCC cells (HN4) were generously provided by Silvio Gutkind (NIH; Bethesda, MD, USA) and cultured in a medium designated for propagation and maintenance, as previously described (Gelbard et al, 2006).

Antibodies. The following primary labelled antibodies were obtained from BD Biosciences (San Diego, CA, USA) for flow cytometry analysis: PE-labelled anti-HER2 (Neu 24.7), anti-EGF receptor 1 (EGFR.1), anti-CD20 (L27), anti-CD56 (B159), FITClabelled anti-CD3 (UCHT1), APC-labelled anti-CD44, and PerCP Cy5.5-labelled anti-CD24. Anti-aldehyde dehydrogenase 1 (ALDH1) antibody and isotype were obtained from LifeSpan Biosciences, Inc. (Seattle, WA, USA). The appropriate isotype controls were purchased from BD Biosciences and R\&D Systems (Minneapolis, MN, USA). The following antibodies were used for western blot analysis: anti- $\beta$-actin (13E5), anti-HER2 (29D8), antiEGFR (D38B1), anti-phospho-NF- $\kappa \mathrm{B}-\mathrm{p} 65$ (93H1), anti-NF- $\kappa \mathrm{B}-$ p65 (C22B4) (Cell Signaling Technology, Danvers, MA, USA), and anti-CD20 (L26) (Santa Cruz Biotechnology, Dallas, TX, USA). Sterile N-acetyl-L-cysteine (Enzo Life Sciences, Farmingdale, NY, USA) was used for ROS inhibition. Both trastuzumab and rituximab (Genentech, San Francisco, CA, USA) were diluted with sterile PBS to a working stock of $2 \mu \mathrm{g} \mu \mathrm{l}^{-1}$.

Radiation treatment. Human tumour cells were harvested while in log-growth phase and kept in suspension during radiation treatment. Cells were irradiated with 5 or $10 \mathrm{~Gy}$ by exposure to a Cs-137 source (Gammacell-1000; AECL/Nordion, Kanata, ON, Canada) at a dose rate of $5.56 \mathrm{~Gy}$ per min.

Flow cytometry. Flow cytometry was used to determine the level of expression of HER2 on breast carcinoma cells (MCF-7, ZR75-1, and MDA-MB-231), EGFR on HNSCC cells (HN4), CD20 on NHL cells (Ramos), and CD3 and CD56 on peripheral blood mononuclear cells (PBMCs) and natural killer (NK) cells. Cells were incubated with antibodies for $30 \mathrm{~min}$ at $4{ }^{\circ} \mathrm{C}$. 7AAD (BD Biosciences) staining was used to determine cell viability. Samples were acquired on a FACScan flow cytometer (Becton Dickinson, Franklin Lakes, NJ, USA) using CellQuest software (BD Biosciences). All cells used for analyses were determined to be viable by $7 \mathrm{AAD}$ staining. A cutoff of $<5 \%$ isotype control staining was used to determine positivity for all samples analysed. Data analysis was performed using FlowJo software (Tree Star, Inc., Ashland, OR, USA). Expression of CD44, CD24, ALDH1, and HER2 on MDA-MB-231 cells was determined as above using an LSRII flow cytometer (Becton Dickinson) and FACS Diva software (BD Biosciences).

Western blot analysis. Cells were lysed with $1 \times$ cell lysis buffer, PMSF, as per manufacturer's instructions (Cell Signaling Technology). Protein concentration was determined using the BCA assay (Thermo Scientific Pierce, Rockford, IL, USA). Equal amounts of protein were loaded per sample and separated by electrophoresis on 4-20\% Tris-glycine gels (Invitrogen, Grand Island, NY, USA) before being transferred to PVDF membranes (Invitrogen) using the iBlot Blotting System (Invitrogen). Densitometric analysis of western blots was performed using the ImageJ application (NIH) to calculate the differences in protein expression (Schneider et al, 2012).

Immunofluorescence. Cells were mock-irradiated or irradiated (10 Gy) and plated on glass coverslips (Fisher Scientific, Pittsburgh, PA, USA). After $48 \mathrm{~h}$ in culture, cells were fixed with ice-cold $\left(-20^{\circ} \mathrm{C}\right)$ methanol (Midland Scientific Inc., Omaha, NE, USA) for $15 \mathrm{~min}$. Cells were stained for HER2 according to the manufacturer's protocol (Cell Signaling Technology). Images were acquired using a Leica DMI 4000B Automated Inverted Microscope (Leica, Buffalo Grove, IL, USA). Fluorescence intensity of individual cells was acquired using ImageJ software (NIH). Corrected total cell fluorescence was calculated as follows: integrated density - (area of selected cell $\times$ mean fluorescence of background), (Gavet and Pines, 2010). For display, contrast was adjusted uniformly across all images.

Reactive oxygen species measurement. Reactive oxygen species were measured using a total ROS detection kit (Enzo Life Sciences) using a protocol modified from Hafer et al (2008). Samples were acquired on a FACScan flow cytometer.

Isolation of natural killer cells. Peripheral blood mononuclear cells obtained from a normal donor and stored at $-80{ }^{\circ} \mathrm{C}$ were thawed, washed, and resuspended in sterile PBS. Natural killer cells were isolated through negative selection with a MACS separator (Miltenyi Biotec, Auburn, CA, USA), according to the manufacturer's protocol. Purity of isolated NK cells was examined by flow cytometry using FITC-CD3, PE-CD56, and appropriate isotype controls (BD Biosciences), and acquired on a FACScan flow cytometer.

Antibody-dependent cell-mediated cytotoxicity assay. Fortyeight hours after irradiation (mock or $10 \mathrm{~Gy}$ ), cells were harvested and labelled with ${ }^{111} \mathrm{In}$ for $30 \mathrm{~min}$ at $37^{\circ} \mathrm{C}$. Radiolabelled tumour cells were incubated with $20 \mu \mathrm{g} \mathrm{ml}^{-1}$ trastuzumab or rituximab for $30 \mathrm{~min}$ on ice. Monoclonal antibody excess was removed by washing. Radiolabelled and mAb-treated tumour cells $\left(2 \times 10^{3}\right)$ suspended in medium were added to 96-well U-bottom plates (Costar, Cambridge, MA, USA) containing increasing numbers of 
effector cells (PBMCs or NK cells) and incubated at $37^{\circ} \mathrm{C}$ with $5 \%$ $\mathrm{CO}_{2}$ for $4 \mathrm{~h}$. Supernatants were collected using cellulose acetate absorption cartridges (Molecular Devices, Sunnyvale, CA, USA) and radioactivity was quantitated using a Cobra Autogamma counter (Packard Instruments, Downers Grove, IL, USA).

Tumour cell proliferation assay. MCF- 7 cells $\left(2 \times 10^{4}\right.$ cells per well) were exposed to radiation (mock or $10 \mathrm{~Gy}$ ), resuspended in medium, and cultured for 4 days in 96-well flat-bottomed plates. After $48 \mathrm{~h}$ of culture, trastuzumab $\left(100 \mu \mathrm{g} \mathrm{ml}^{-1}\right)$, rituximab $\left(100 \mu \mathrm{g} \mathrm{ml}^{-1}\right)$, or medium was added to triplicate wells. ${ }^{3} \mathrm{H}$ thymidine $(1 \mu \mathrm{Ci}$ per well) was added to all wells for the last $24 \mathrm{~h}$ of culture, and cells were harvested using a Tomtec cell harvester (Wallac Inc., Gaithersburg, MD, USA). The incorporated radioactivity was measured using a Wallac 1205 Betaplate liquid scintillation counter (Wallac). All culture steps were performed at $37^{\circ} \mathrm{C}$ with $5 \% \mathrm{CO}_{2}$.

Statistical analysis. Significant differences in the distribution of data acquired by flow cytometry analysis were determined by the Kolmogorov-Smirnov test using FloJo software (Tree Star, Inc.). Statistical differences between two treatments were analysed by unpaired Student's $t$-test with a two-tailed distribution and
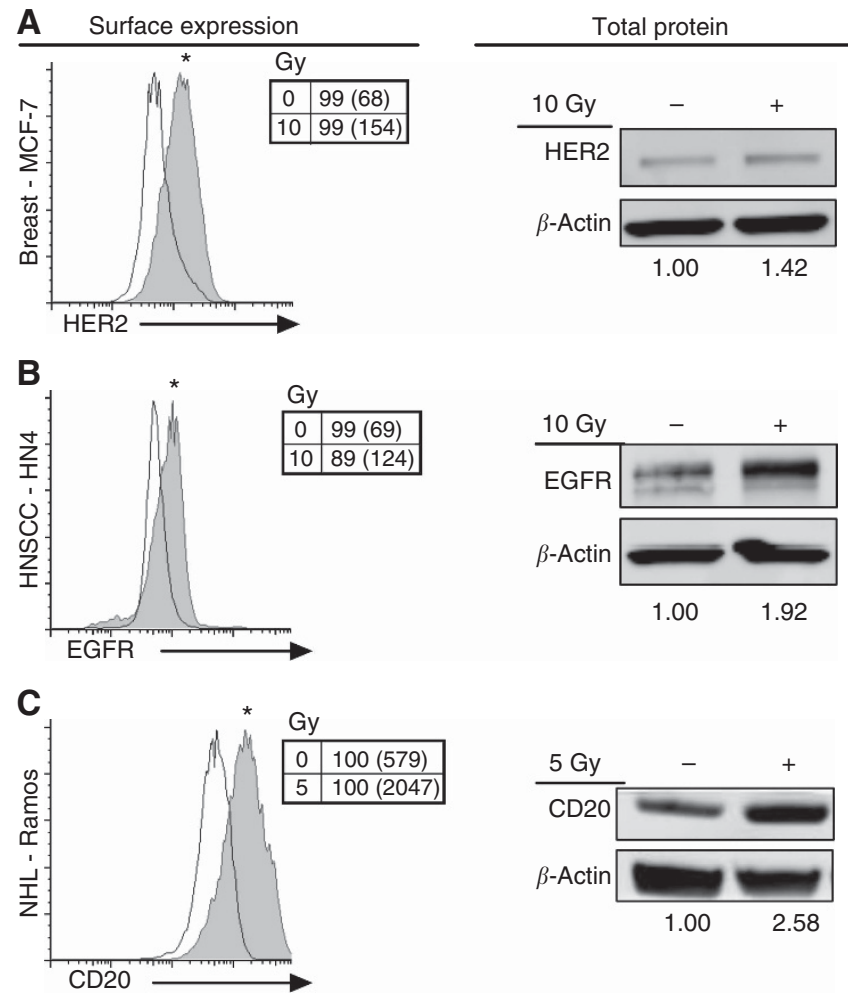

Figure 1. Radiation increases total and cell-surface expression of target molecules. Tumour-cell lines were mock-irradiated (open histograms) or treated with radiation (shaded histograms). After $48 \mathrm{~h}$ of culture, expression of target molecules was examined by flow cytometry and western blot analysis. (A) Expression of HER2 in MCF-7 breast carcinoma cells. (B) Expression of EGFR in HN4 HNSCC cells. (C) Expression of CD20 in Ramos NHL cells. Insets: numbers indicate \% positive cells and MFI (parentheses). Cells used for analysis were viable as determined by 7AAD staining, and staining was corrected for isotype control binding. This experiment was repeated two times with similar results. Comparisons of protein expression HER2 (A), EGFR (B), or CD20 (C) were determined by using band densitometry analysis using ImageJ software normalising $\beta$-actin relative to control. ' ${ }^{* \prime}$ denotes statistical significance relative to untreated cells $(P<0.05)$. reported as $P$-values using GraphPad Prism 6 for Mac OS $\mathrm{X}$ (GraphPad Software Inc., La Jolla, CA, USA).

\section{RESULTS}

Radiation increases total and cell-surface expression of $\mathrm{mAb}$ target molecules. As radiation was used in these studies to modify surface phenotype of tumour cells as opposed to model a therapeutic setting, we focused on a single fraction of radiation. We first examined whether single-dose radiation could modulate
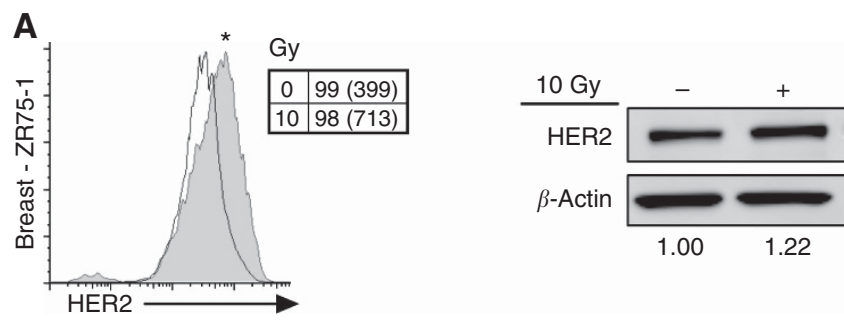

B

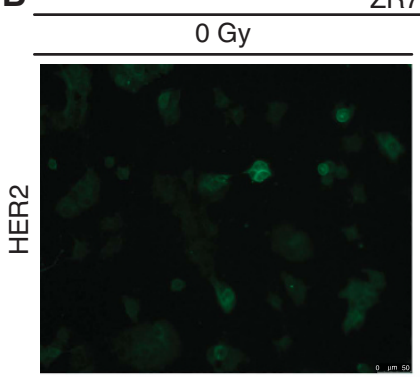
ZR75-1
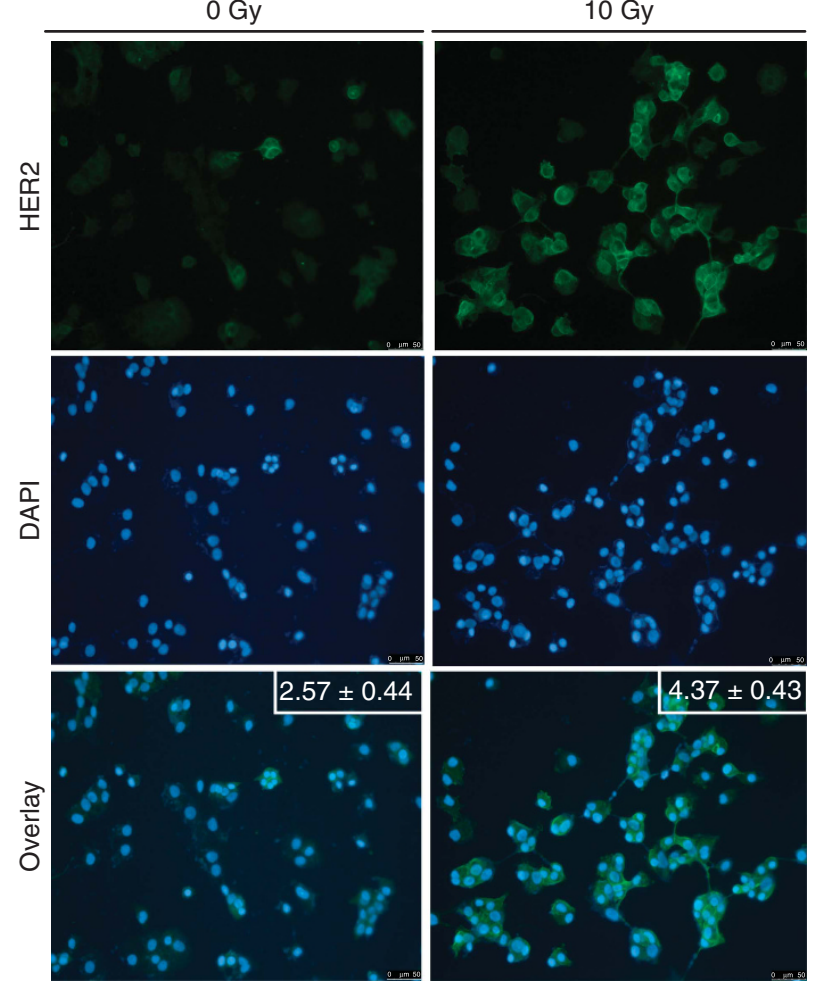

Figure 2. Radiation-induced upregulation of HER2 cell-surface expression visualised by immunofluorescence. (A) ZR75-1 breast carcinoma cells were mock irradiated (open histograms) or irradiated with $10 \mathrm{~Gy}$ (shaded histograms), and HER2 expression was examined by flow cytometry and western blot $48 \mathrm{~h}$ post exposure. Insets: numbers indicate \% positive cells and MFI (parentheses). Cells used for analysis were viable as determined by 7AAD staining, and staining was corrected for isotype control binding. ' ${ }^{\star \prime}$ denotes statistical significance relative to untreated cells $(P<0.05)$. (B) ZR75-1 cells were mock irradiated or irradiated with $10 \mathrm{~Gy}$, and HER2 expression was examined by immunofluorescence $48 \mathrm{~h}$ post exposure. Nuclei were stained with DAPI; images represent $\times 20$ magnification. Insets: average HER2 expression per cell corrected by total cell fluorescence \pm s.d. The images presented are representative of three independent experiments with similar results. 
$\mathrm{mAb}$ target expression in breast (MCF-7), HNSCC (HN4), and NHL (Ramos) cell lines. Forty-eight hours after cells were exposed to radiation (mock or $10 \mathrm{~Gy}$ ), protein expression was determined by flow cytometry and western blot analysis (Figure 1A). Exposing MCF-7 cells to radiation significantly increased cell-surface expression of HER2 (2.3-fold; $P<0.01)$ and total expression of HER2 (1.4-fold). In the HN4 cell line, radiation significantly upregulated cell-surface expression of EGFR (1.8-fold; $P<0.01$ ), and total EGFR increased by 1.9-fold (Figure 1B). Radiation (mock or $5 \mathrm{~Gy}$ ) of the NHL (Ramos) cell line significantly increased cellsurface expression of CD20 (3.5-fold; $P<0.01$ ), and total protein increased by 2.6 -fold as measured by flow cytometry and western blot analysis, respectively (Figure 1C). 7AAD staining confirmed that radiation doses were sublethal $(7 \mathrm{AAD} \%$ positive $<10 \%)$. In another experiment, ZR75-1 breast cancer cells exposed to radiation showed a significant $(1.8$-fold; $P<0.01)$ increase in HER2 cell-surface expression and a 1.2-fold increase in total HER2 (Figure 2A and B). We used immunofluorescence microscopy to confirm subcellular localisation of HER2 protein post radiation. ZR75-1 cells were irradiated (mock or 10 Gy) and cultured for $48 \mathrm{~h}$ before immunofluorescence analysis. Fluorescence intensity of individual cells was quantified using ImageJ (NIH). Treated cells showed a significant (1.7-fold; $P=0.0084)$ increase in mean HER2corrected total cellular fluorescence compared with mock-irradiated cells (Figure 2A). In all cases, viability of tumour cells after radiation exposure remained above $90 \%$. Collectively, these data show that radiation treatment leads to significant upregulation of the mAb targets HER2, EGFR, and CD20 at both cell-surface and total protein levels.

Radiation increases HER2 expression in triple-negative breast cancer cells. Triple-negative breast cancer (TNBC) is defined by the absence of oestrogen receptor, progesterone receptor, and HER2 expression, as characterised by IHC (Stagg and Allard, 2013). To determine whether radiation could upregulate cellsurface HER2 expression on a TNBC cell line, we exposed MDAMB-231 cells to $10 \mathrm{~Gy}$. Forty-eight hours post exposure, HER2 expression had significantly increased in irradiated cells (1.4-fold; $P<0.05)$ compared with mock-irradiated cells (Figure $3 \mathrm{~A}$ ), as determined by flow cytometry. We also examined the resident

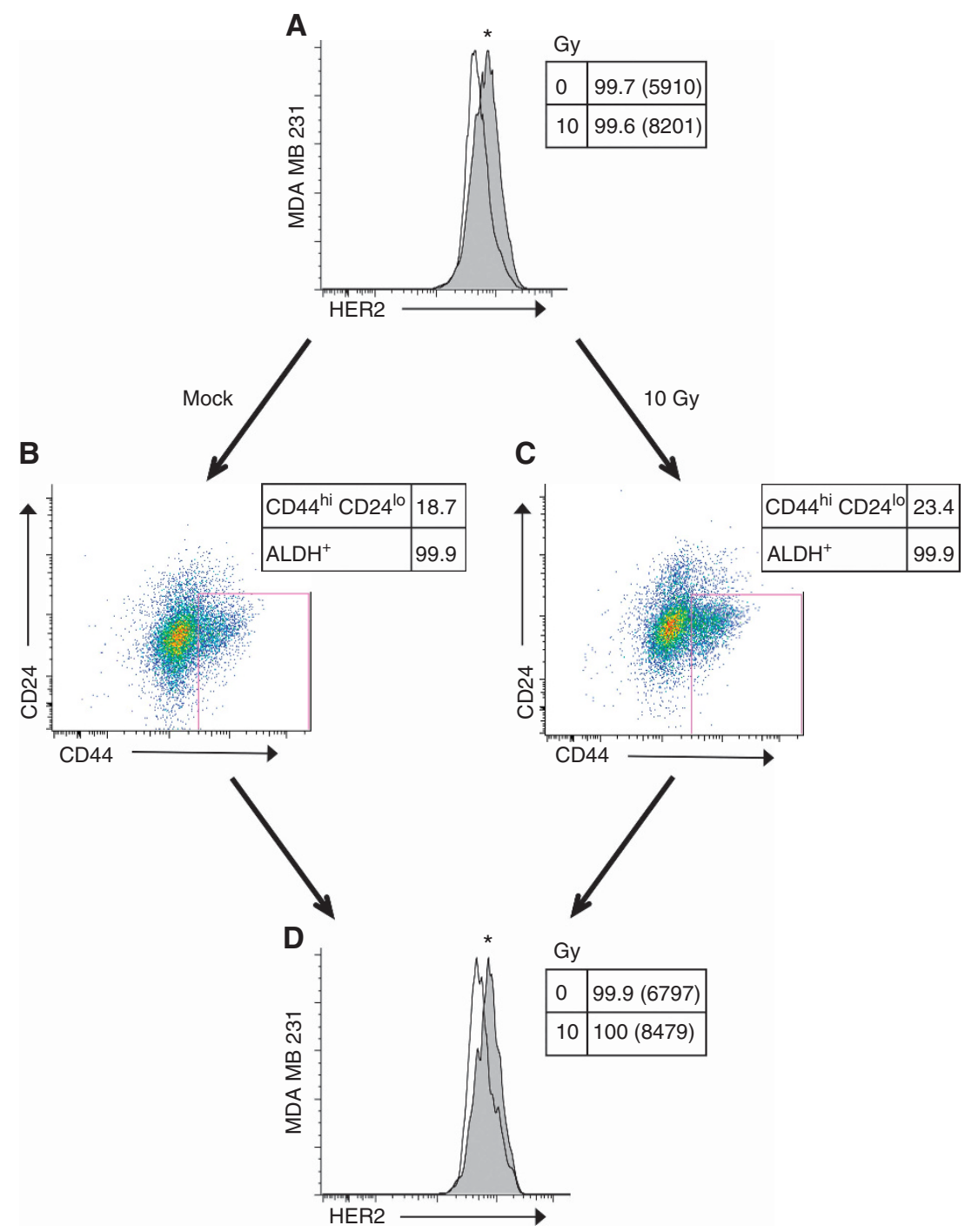

Figure 3. Radiation increased HER2 expression in TNBC cells. (A) The TNBC cell line MDA-MB-231 was mock irradiated (open histograms) or irradiated with $10 \mathrm{~Gy}$ (shaded histograms), and HER2 expression was examined by flow cytometry $48 \mathrm{~h}$ post exposure. Inset: Numbers indicate \% positive cells and MFI (parentheses). (B, C) Stem-cell populations of (B) mock-irradiated and (C) irradiated (10 Gy) MDA-MB-231 cells were examined by flow cytometry. Insets: numbers indicate \% positive cells. (D) HER2 expression of gated MDA-MB-231 stem-cell populations, as shown in B, C respectively, that were mock-irradiated (open histograms) or irradiated with 10 Gy (shaded histograms). Inset: numbers indicate \% positive cells and MFI (parentheses). ' ${ }^{\star \prime}$ denotes statistical significance relative to untreated cells $(P<0.05)$. 
stem-cell population in MDA-MB-231 cells (defined as $\mathrm{CD} 44^{\mathrm{hi}} \mathrm{CD} 24^{\mathrm{lo}} \mathrm{ALDH} 1^{+}$) by flow cytometry. The proportion of resident stem cells had increased in MDA-MB-231 cells treated with $10 \mathrm{~Gy}$ (Figure 3B) compared with mock-irradiated cells (Figure 3C) owing to resident stem cells' relative radioresistance, as previously reported (Phillips et al, 2006). However, radiation did significantly upregulate HER2 in this cell population (Figure 3D).

Radiation-induced HER2 upregulation is time- and dose dependent. We next investigated the role of dose and time in radiation-induced upregulation of HER2. We exposed MCF-7 breast cancer cells to $10 \mathrm{~Gy}$ and examined HER2 expression daily up to $96 \mathrm{~h}$ (Figure 4A). HER2 was significantly upregulated $(P<0.01)$ at the cell surface $24 \mathrm{~h}$ post irradiation and continued to increase up to $96 \mathrm{~h}$ (Figure $4 \mathrm{~A}$ ). To explore the effect of radiation dose, we exposed MCF-7 cells to a single $5 \mathrm{~Gy}$ dose of radiation and analysed HER2 cell-surface expression over time up to $96 \mathrm{~h}$ post irradiation (Figure 4B). Results showed that even at this lower dose of radiation, HER2 expression was significantly upregulated $(P<0.01) 24 \mathrm{~h}$ post treatment and continued to increase for up to $96 \mathrm{~h}$ (Figure 4B).

Radiation-induced upregulation of HER2 is mediated by ROS and is associated with phosphorylation of NF- $\kappa$ B. It has previously been shown that NF- $\kappa \mathrm{B}$ is activated by ROS, including ROS secondary to ionising radiation (Gloire et al, 2006). We used the ROS inhibitor $\mathrm{N}$-acetyl-L-cysteine to associate radiationinduced ROS with HER2 cell-surface upregulation (Figure 5). We first confirmed the response of ROS to radiation and the ability of the ROS inhibitor to abrogate ROS production. Radiation $(10$ Gy) significantly increased ROS ( 1.5 -fold; $P<0.01$ ); however,

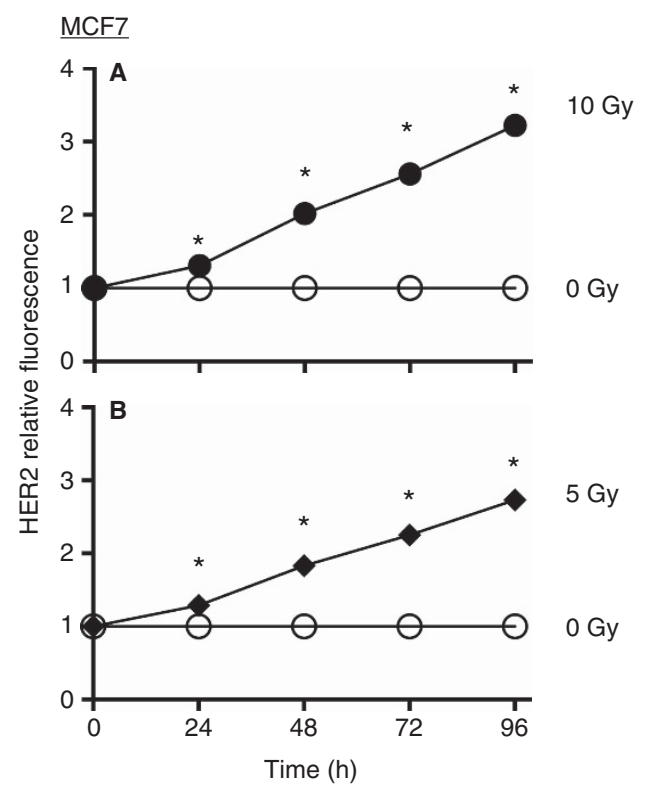

Figure 4. Radiation-induced HER2 upregulation is time- and dose dependent. (A) MCF-7 cells were mock-irradiated (open circles) or irradiated with $10 \mathrm{~Gy}$ (closed circles). After treatment, cells were harvested daily up to $96 \mathrm{~h}$ and examined for HER2 expression by flow cytometry. (B) MCF-7 cells were mock-irradiated (open circles) or irradiated with $5 \mathrm{~Gy}$ (closed diamonds). After treatment, cells were harvested daily up to $96 \mathrm{~h}$ and examined for HER2 expression by flow cytometry. Relative fluorescence was calculated as treated sample $\mathrm{MFI} \div$ control sample MFI at each time point. ' ${ }^{\prime \prime}$ denotes statistical significance relative to mock-irradiated cells at each 24-h time point $(P<0.05)$. This experiment was repeated three additional times with similar results. this post-irradiation increase was significantly inhibited $(P<0.01)$ when $10 \mathrm{~Gy}$ was given in the presence of ROS inhibitor (Figure $5 \mathrm{~A}$ and $\mathrm{B}$ ). To determine whether alterations in intracellular ROS were associated with changes in HER2 cell-surface expression, we mockirradiated or irradiated (10 Gy) MCF-7 cells in the presence or absence of ROS inhibitor. Forty-eight hours post treatment, HER2 cell-surface expression was analysed by flow cytometry. Cells exposed to radiation ( $10 \mathrm{~Gy}$ ) alone showed a two-fold increase in HER2 expression, whereas cells irradiated (10 Gy) in the presence of ROS inhibitor showed only a 1.4-fold increase in HER2 expression, a significant inhibition of HER2 upregulation compared with radiation treatment alone $(P<0.01)$ (Figure 5A and $C$ ). These data suggest that increased levels of intracellular ROS mediate radiation-induced HER2 upregulation.

NF- $\kappa \mathrm{B}$, a family of transcription factors activated by a variety of extracellular stimuli, can induce expression of a large number of genes, including those necessary for survival and proliferation (Diamant and Dikstein, 2013). We used phospho-NF- $\kappa$ B-p65 (Ser536) as a marker of NF- $\kappa$ B activation. We exposed MCF-7 cells to radiation (mock or $10 \mathrm{~Gy}$ ) in the presence or absence of ROS inhibitor and analysed phospho-NF- $\kappa$ B-p65 levels by western blot $24 \mathrm{~h}$ post treatment. Radiation (10 Gy) induced a significant increase $(2.2- \pm 0.07$-fold; $P=0.0036)$ in phosphorylation of the p65 subunit (Ser536) (Figure 5D). When cells were irradiated in the presence of ROS inhibitor, phosphorylation was not significant $(P=0.69)$ compared with baseline, and was significantly lower $(P=0.0371)$ than the phospho-NF- $\kappa \mathrm{B}-\mathrm{p} 65$ level seen in cells treated with radiation alone (Figure $5 \mathrm{D}$ ).

Radiation enhances tumour cell killing and antiproliferative effects of trastuzumab. We used an in vitro assay for ADCC to analyse the functional effects of a radiation-induced increase in HER2 expression. We initially used normal-donor PBMCs as effector cells at decreasing effector:target ratios (100:1 to $25: 1)$. Flow cytometry revealed the $\mathrm{NK}$ cell $\left(\mathrm{CD} 6^{+} / \mathrm{CD}^{-}\right)$subpopulation of normal-donor PBMCs to be 19\% (Figure 6A). Forty-eight hours after radiation exposure (mock or $10 \mathrm{~Gy}$ ), MCF-7 target cells were left untreated, incubated with trastuzumab $\left(20 \mu \mathrm{g} \mathrm{ml}^{-1}\right)$ or incubated with isotype control antibody (rituximab), and then incubated with effector cells. Mock-irradiated cells incubated with trastuzumab showed significantly increased sensitivity to cytotoxicity compared with mock-irradiated cells left untreated $(P=0.0022)$ or incubated with isotype control antibody $(P=0.0011)$, confirming previous findings that trastuzumab could mediate ADCC in HER2-nonamplified cell lines. MCF-7 cells exposed to radiation ( $10 \mathrm{~Gy}$ ) showed significant increases in sensitivity to ADCC of 1.4-fold at 100:1 $(P=0.0031)$ and 1.3 -fold at 50:1 $(P=0.0067)$ effector:target ratios compared with mockirradiated cells incubated with trastuzumab (Figure 6A).

To further investigate the importance of NK cell activity, we purified NK cells from normal-donor PBMCs and used them as effector cells at decreasing effector:target ratios $(25: 1$ to $1.56: 1)$. Flow cytometry placed NK cell $\left(\mathrm{CD} 6^{+} / \mathrm{CD}^{-}\right)$purification at $94 \%$ (Figure 6B). The sensitivity to cytotoxicity of irradiated (10 Gy) MCF-7 cells incubated with trastuzumab significantly increased (two-fold) compared with untreated cells incubated with trastuzumab at 4 effector:target ratios $(P<0.0001$ at effector:target ratio of $25: 1$ ) (Figure $6 \mathrm{~B}$ ). These data collectively indicate that radiation exposure sensitises tumour cells to trastuzumab-mediated ADCC, and that NK cells appear to have the major role in ADCC, although other innate immune cells have been implicated as effectors.

Trastuzumab exerts tumour control by a variety of mechanisms, including inhibition of proliferation (Tagliabue et al, 2012). We investigated the sensitivity of MCF-7 cells to the antiproliferative actions of trastuzumab before and after radiation treatment. We irradiated MCF-7 cells (mock or 10Gy) and measured proliferation by ${ }^{3} \mathrm{H}$-thymidine incorporation 4 days post 
A

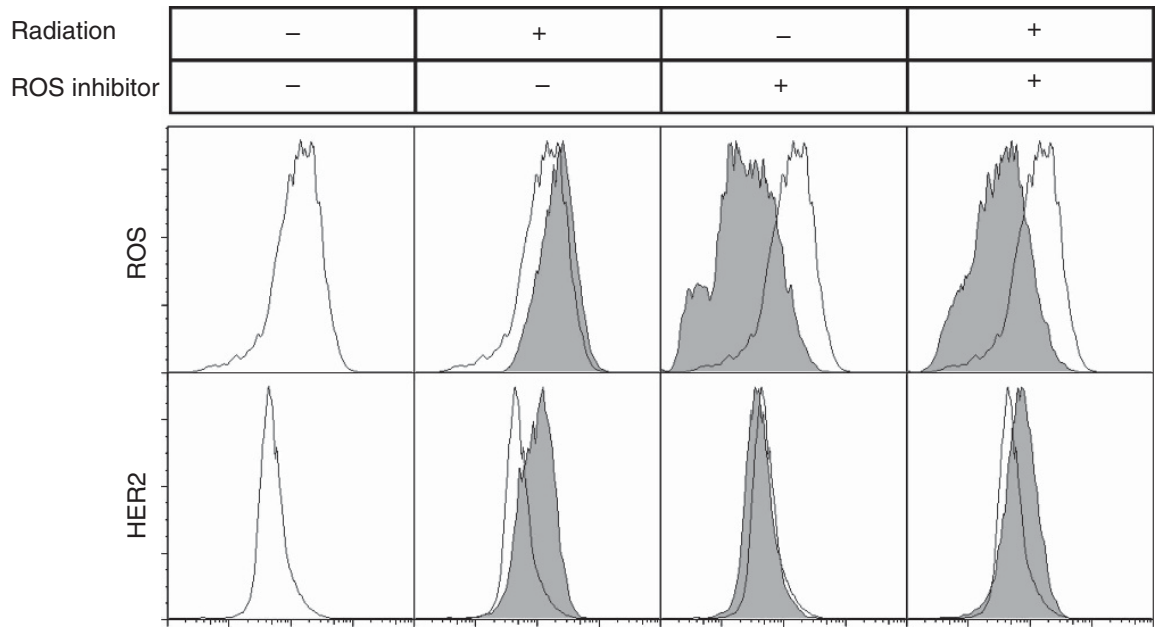

B
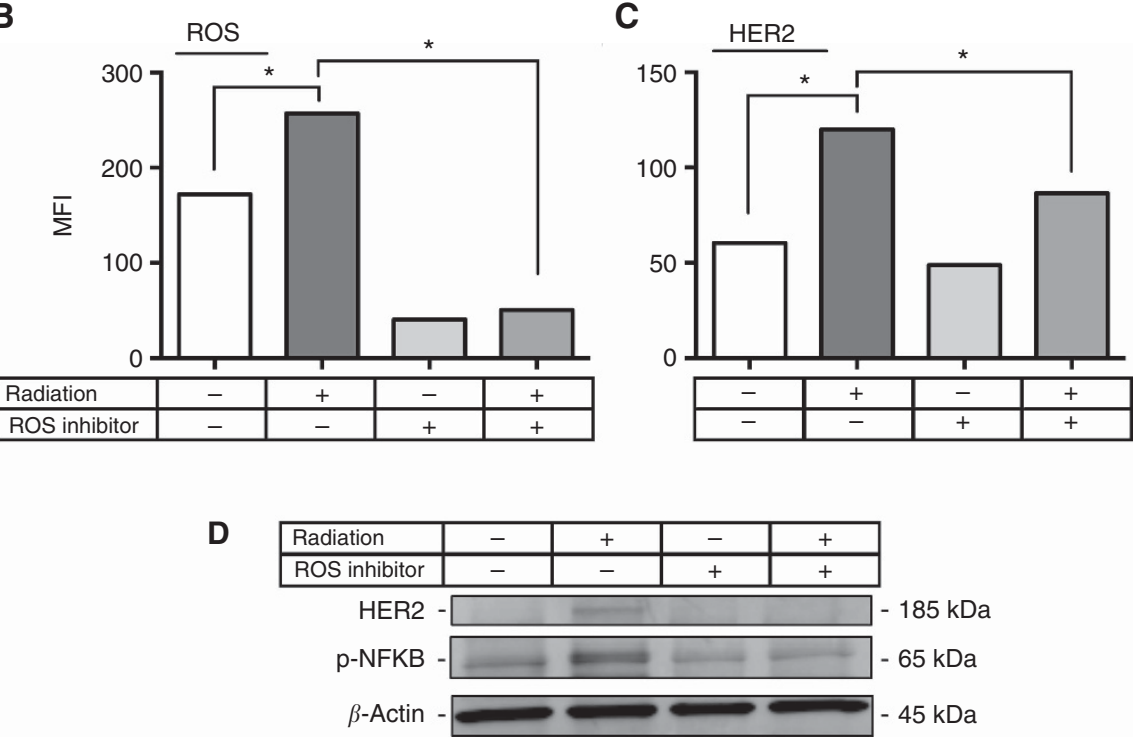

Figure 5. Radiation-induced HER2 upregulation is mediated by ROS and associated with phosphorylation of NF- $\kappa$ B. (A) Intracellular ROS and cell-surface HER2 were measured by flow cytometry. MCF-7 cells were mock irradiated (open histograms), irradiated with 10 Gy (shaded histograms), treated with ROS inhibitor (N-acetyl-L-cysteine) (shaded histograms), or irradiated with $10 \mathrm{~Gy}$ in the presence of ROS inhibitor (shaded histograms). (B) MFI of ROS measurements taken from histograms in A. (C) MFI of HER2 cell-surface expression taken from histograms in A. (D) MCF-7 cells were mock irradiated, irradiated with $10 \mathrm{~Gy}$, treated with ROS inhibitor, or irradiated with $10 \mathrm{~Gy}$ in the presence of ROS inhibitor. Expression of HER2, phospho-NF- $\kappa$ B-p65, and $\beta$-actin was examined by western blot analysis $24 \mathrm{~h}$ post treatment. ' ${ }^{\prime \prime}$ denotes statistical significance $(P<0.05)$. The data presented is representative of three independent experiments with similar results.

treatment. Trastuzumab $\left(100 \mu \mathrm{g} \mathrm{ml}^{-1}\right)$ or isotype control antibody $\left(100 \mu \mathrm{g} \mathrm{ml}^{-1}\right)$ was added $48 \mathrm{~h}$ post treatment to allow upregulation of HER2. Mock-irradiated MCF-7 cells showed no sensitivity to trastuzumab $(P=0.068)$. However, proliferation of irradiated $(10 \mathrm{~Gy})$ cells cultured in the presence of trastuzumab was significantly reduced compared with irradiated (10 Gy) cells cultured alone $(P=0.049)$ (Figure $6 \mathrm{C})$. These data suggest that MCF-7 cells that are initially insensitive to the antiproliferative effects of trastuzumab are sensitised by radiation pretreatment.

\section{DISCUSSION}

Monoclonal antibodies for therapy have transformed oncology; however, the prevailing dogma has been that expression of the 'target' must be present for the mAb to be effective. Our study was designed to ascertain whether ionising radiation can upregulate poorly expressed targets and thus enlarge the opportunities for therapeutic mAbs. HER2, a receptor tyrosine kinase that mediates cell proliferation and survival, is overexpressed by $15-23 \%$ of breast cancers (Mohd Sharial et al, 2012). EGFR is a related receptor tyrosine kinase that is overexpressed by $90 \%$ of HNSCC (Tejani et al, 2010). CD20 is expressed by B-cell lymphomas that represent $85 \%$ of all NHL (Dotan et al, 2010). The FDA has approved an $\mathrm{mAb}$ therapy for each of these three target molecules: trastuzumab for HER2, cetuximab for EGFR, and rituximab for CD20 (Scott et al, 2012). Although mAb therapy has had some clinical success, issues with resistance have limited its efficacy. Up to $70 \%$ of patients eligible to receive trastuzumab will not respond to treatment (Kute et al, 2004). For patients with recurrent or metastatic disease, the response rate to cetuximab monotherapy is 13\% (Markovic and Chung, 2012). Finally, one-third of patients with diffuse large B-cell lymphoma will develop relapsed/refractory disease despite the availability of rituximab (Friedberg, 2011). For these patients, the goal is to improve outcomes by delivering 


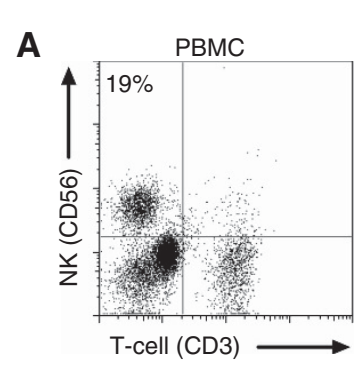

Effector
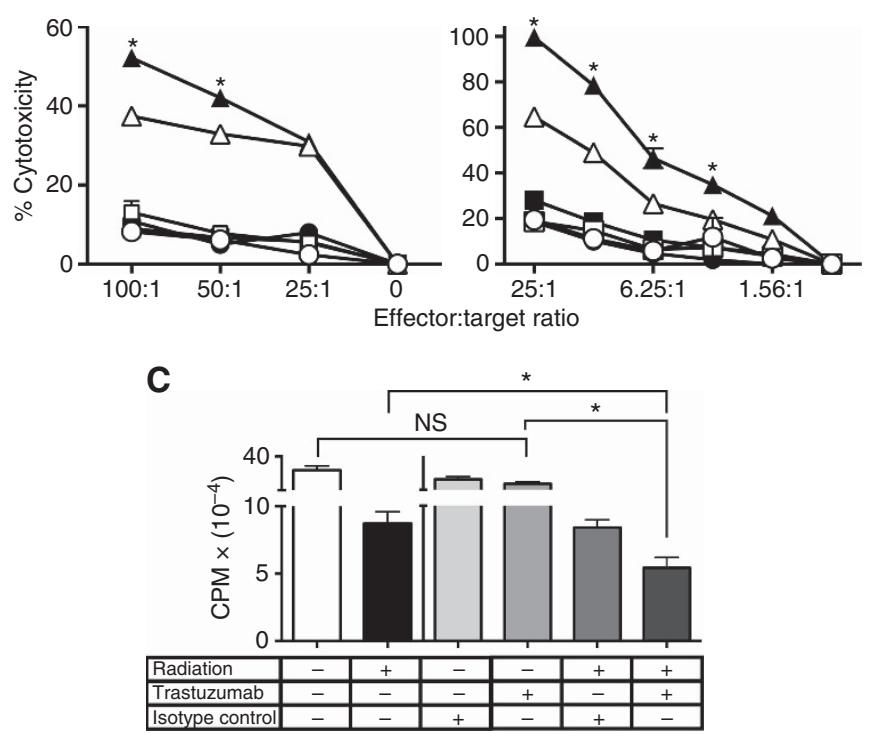

Figure 6. Radiation exposure augments tumour-cell killing in an in vitro ADCC assay and sensitises tumour cells to the antiproliferative effects of trastuzumab. (A) Peripheral blood mononuclear cells isolated from a normal donor were used as effector cells. Number indicates percent NK cells in sample. MCF-7 cells were mock irradiated or irradiated with $10 \mathrm{~Gy} 48 \mathrm{~h}$ before the ADCC assay. Triplicate wells of untreated cells (open symbols) and irradiated cells (closed symbols) were incubated with trastuzumab (triangles), isotype control antibody (rituximab) (circles), or left untreated (squares). (B) Natural killer cells were purified from the normal-donor PBMCs used in $\mathbf{A}$ and used as effector cells. Number indicates NK cell purity. MCF-7 cells were mockirradiated or irradiated with $10 \mathrm{~Gy} 48 \mathrm{~h}$ before the ADCC assay. Three replicate wells of untreated cells (open symbols) and irradiated cells (closed symbols) were incubated with trastuzumab (triangles), incubated with isotype control antibody (rituximab) (circles), or left untreated (squares). ' ${ }^{\star \prime}$ denotes statistical significance relative to untreated cells incubated with trastuzumab $(P<0.05)$. (C) MCF-7 cells were irradiated (mock or $10 \mathrm{~Gy}$ ) and cultured in triplicate wells of a 96well plate for 4 days. Trastuzumab or isotype control antibody (rituximab) was added for the final $48 \mathrm{~h}$ of culture. Proliferation was then measured by incorporation of ${ }^{3} \mathrm{H}$-thymidine, which was added for the final $24 \mathrm{~h}$ of culture. The data presented is representative of three independent experiments with similar results. Data were analysed using a non-paired Student's t-test. ' ${ }^{\star \prime}$ denotes statistical significance $(P<0.05)$.

a single dose of radiation to upregulate respective target molecules before mAb therapy. This approach has been tried before. Kitazono et al (2001) previously reported using a low-dose sensitising agent (specifically, a histone deacetylase inhibitor) to upregulate a specific molecular target to induce expression of a functional $\mathrm{Na} / \mathrm{I}$ transporter in thyroid cancer cells to improve the targeting of ${ }^{125} \mathrm{I}$.

Here, we first showed that radiation upregulates HER2, EGFR, and CD20 (Figures 1-3). We used trastuzumab and its target HER2 as a model to investigate the potential of radiation to upregulate targets of $\mathrm{mAb}$ therapy. We showed that radiation upregulates HER2 in 3 out of 3 breast cancer cell lines tested (MCF-7, ZR75-1, and MDA-MB-231) (Figures 1-3). Although trastuzumab is currently indicated for breast cancer that is HER2 $3^{+}$by IHC, it has been shown to mediate ADCC in breast cancer cells that do not express high levels of HER2 (Beano et al, 2008; Collins et al, 2012). We suggest that radiation may increase the efficacy of trastuzumab in breast cancer that is $0-2^{+}$HER2 by IHC at diagnosis and thus ineligible for $\mathrm{mAb}$ therapy. Triple-negative breast cancer, which lacks oestrogen receptor, progesterone receptor, and HER2 expression, does not currently benefit from HER2-targeted therapy or endocrine therapy (Stagg and Allard, 2013). However, in our study, radiation increased HER2 expression on the TNBC cell line MDA-MB-231 (Figure 3A). This is a similar upregulation as that seen in HER2 ${ }^{+}$cell lines (Figures 1A and 2A), although comparisons in expression of HER2 cannot be made between the cell lines because the data was acquired on different flow cytometers. As previously reported, radiation can transiently increase the proportion of cancer stem cells within a tumour because radiation eradicates the non-stem-cell population, whereas cancer stem cells are relatively radioresistant (Phillips et al, 2006; Lagadec et al, 2010; Pajonk et al, 2010). We observed this phenomenon (Figure $3 \mathrm{~B}$ and $\mathrm{C}$ ) while noting that radiation was capable of significantly upregulating HER2 expression on this resident stem-cell population (Figure 3D), suggesting that radiation may have clinical benefit in TNBC by making tumours and particularly cancer stem cells more sensitive to HER2-targeted therapy as has been previously proposed (Ithimakin et al, 2013).

We next explored the longevity of post-radiation HER2 expression and demonstrated that HER2 upregulation was maintained up to $96 \mathrm{~h}$ after exposure to 5-10 Gy (Figure 4). Interestingly, HER2 upregulation was not substantially different after doses of 5 or $10 \mathrm{~Gy}$ at all time points examined. We suggest that, in vivo, fractionated doses of radiation as low as 5 Gy may provide an effect similar to a single dose of $10 \mathrm{~Gy}$ while limiting the potential for toxicity to normal tissue. Our data confirm those of Voutsas et al (2013) who have reported that radiation of two breast cancer cell lines with 5 Gy induced HER2 gene amplification, and we extend these observations into additional targets for $\mathrm{mAb}$ therapy, EGFR and CD20.

We next examined the potential mechanism of radiationinduced HER2 upregulation. As previously reported (Gloire et al, 2006), ROS generated by irradiation mediates NF- $\kappa$ B activation. Cao et al (2009) showed that following radiation, NF- $\kappa$ B subunits p65 and p50 are recruited to the HER2 promoter. We confirmed and extended these findings by demonstrating the link with HER2 expression. Using a ROS inhibitor, we showed that radiationinduced ROS is required for the upregulation of HER2 and correlated this finding with p65 phosphorylation, a marker of NF$\kappa \mathrm{B}$ activation (Figure 5) (Diamant and Dikstein, 2013). It is interesting to note that other standard-of-care therapies for breast cancer, such as doxorubicin and docetaxel, have been reported to induce ROS, suggesting that these therapies could also augment mAb therapy (Tsang et al, 2003; Cao et al, 2004).

Regarding the functional consequence of enhanced HER2 expression on tumour cells, we found that upregulation of HER2 correlated with the ability of innate immune effector cells to lyse tumour cells incubated with trastuzumab through the action of ADCC, an important mechanism by which trastuzumab functions (Figure 6A and B) (Weiner et al, 2009). Radiation alone did not increase the sensitivity of MCF-7 cells to ADCC, suggesting that the increase in cell lysis observed when radiation was combined with trastuzumab was trastuzumab, and therefore HER2, dependent. Evidence suggests that trastuzumab prevents HER2 signaling, thus inhibiting proliferation (Tagliabue et al, 2012). MCF-7 cells have previously been shown to be resistant to the antiproliferative actions of trastuzumab (Junttila et al, 2009). Although we 
confirmed this finding, we also showed that radiation sensitises MCF-7 cells to trastuzumab-mediated inhibition of proliferation (Figure 6C). Radiation alone reduces the proliferation capacity of these cells, but this effect is significantly amplified by the addition of trastuzumab as has been seen previously (Duru et al, 2012).

Strategies for sensitising tumours to ADCC by upregulating target molecules have previously relied on systemic therapies. Maruyama et al (2011) showed that lapatinib upregulates HER2, thus improving trastuzumab-mediated ADCC. Similarly, Shimizu et al (2010) used a histone deacetylase inhibitor to sensitise B-cell lymphoma to rituximab therapy through upregulation of CD20. We suggest that localised radiation therapy may avoid the toxicities associated with systemic therapy.

As a mechanism for ADCC-induced antitumor adaptive T-cell response, Weiner et al (2009) proposed that NK cell-mediated tumour destruction liberates TAAs that are then taken up by dendritic cells and presented to $\mathrm{CD} 8{ }^{+}$and $\mathrm{CD} 4^{+} \mathrm{T}$ cells. These liberated TAAs would include the targeted antigen as well as other antigens expressed by the tumour. The T-cell response would thus be more diverse and would confer continued protection against primary tumour recurrence and distant metastasis after the primary response of ADCC was complete. We suggest that irradiation of a single lesion, in combination with mAb therapy, may invoke NK cell-mediated tumour destruction, release TAAs, and generate an adaptive immune response that would attack distant metastatic lesions.

Recent advances, including the development of new $\mathrm{mAb}$ that target HER2, are providing numerous opportunities for combining radiation with targeted therapy. In 2013, the FDA-approved adotrastuzumab emtansine (Kadcyla; Genentech), the first antibodydrug conjugate, for the treatment of HER2 ${ }^{+}$metastatic breast cancer. Ado-trastuzumab emtansine, which is trastuzumab linked to maytansinoid DM1 (ImmunoGen) (Reichert, 2013), has been shown to induce ADCC, and thus could provide the same NK cellmediated tumour killing as trastuzumab while also delivering a cytotoxic agent (Barok et al, 2011). Multiple novel antibody-drug conjugates are under development, including some that use shigalike toxin $1 \mathrm{~A}$, a ribosome inhibitor, conjugated with $\mathrm{mAb}$ against either HER2 or CD20 (Rajagopalan et al, 2013; Wirth et al, 2013).

Here, we demonstrate that single-dose radiation enhances $\mathrm{mAb}$ therapy. This finding offers a new therapeutic combination to cancer patients, expands the patient population eligible for targeted therapy, and elucidates a mechanism for using radiation in combination with immunotherapy.

\section{ACKNOWLEDGEMENTS}

We thank Dr Jeffrey Schlom for helpful suggestions, Marion Taylor for excellent technical assistance, and Bonnie L Casey for editorial assistance in the preparation of this manuscript. This research was supported by the Intramural Research Program of the Center for Cancer Research, National Cancer Institute, and National Institutes of Health. Further support was provided by the NIH Medical Research Scholars Program, a public-private partnership supported by the NIH along with generous contributions to the Foundation for the NIH from Pfizer Inc., The Leona M and Harry B Helmsley Charitable Trust, the Howard Hughes Medical Institute, and other private donors. For a complete list of donors, see the Foundation website at http://www.fnih.org/work/programsdevelopment/medical-research-scholars-program.

\section{CONFLICT OF INTEREST}

The authors declare no conflict of interest.

\section{REFERENCES}

Barok M, Tanner M, Koninki K, Isola J (2011) Trastuzumab-DM1 causes tumour growth inhibition by mitotic catastrophe in trastuzumab-resistant breast cancer cells in vivo. Breast Cancer Res 13(2): R46.

Beano A, Signorino E, Evangelista A, Brusa D, Mistrangelo M, Polimeni MA, Spadi R, Donadio M, Ciuffreda L, Matera L (2008) Correlation between NK function and response to trastuzumab in metastatic breast cancer patients. J Transl Med 6: 25.

Cao DX, Qiao B, Ge ZQ, Yuan YJ (2004) Comparison of burst of reactive oxygen species and activation of caspase- 3 in apoptosis of K562 and HL-60 cells induced by docetaxel. Cancer Lett 214(1): 103-113.

Cao N, Li S, Wang Z, Ahmed KM, Degnan ME, Fan M, Dynlacht JR, Li JJ (2009) NF-kappaB-mediated HER2 overexpression in radiation-adaptive resistance. Radiat Res 171(1): 9-21.

Chakraborty M, Abrams SI, Camphausen K, Liu K, Scott T, Coleman CN, Hodge JW (2003) Irradiation of tumor cells up-regulates Fas and enhances CTL lytic activity and CTL adoptive immunotherapy. JI Immunol 170(12): 6338-6347.

Chakraborty M, Abrams SI, Coleman CN, Camphausen K, Schlom J, Hodge JW (2004) External beam radiation of tumors alters phenotype of tumor cells to render them susceptible to vaccine-mediated T-cell killing. Cancer Res 64(12): 4328-4337.

Collins DM, O'Donovan N, McGowan PM, O'Sullivan F, Duffy MJ, Crown J (2012) Trastuzumab induces antibody-dependent cell-mediated cytotoxicity (ADCC) in HER-2-non-amplified breast cancer cell lines. Ann Oncol 23(7): 1788-1795.

Diamant G, Dikstein R (2013) Transcriptional Control by NF-kappaB: Elongation in Focus. Biochim Biophys Acta 1829(9): 937-945.

Dotan E, Aggarwal C, Smith MR (2010) Impact of rituximab (Rituxan) on the treatment of B-cell non-hodgkin's lymphoma. $P$ \& $T$ 35(3): 148-157.

Duru N, Fan M, Candas D, Menaa C, Liu HC, Nantajit D, Wen Y, Xiao K, Eldridge A, Chromy BA, Li S, Spitz DR, Lam KS, Wicha MS, Li JJ (2012) HER2-associated radioresistance of breast cancer stem cells isolated from HER2-negative breast cancer cells. Clin Cancer Res 18(24): 6634-6647.

Formenti SC, Demaria S (2013) Combining radiotherapy and cancer immunotherapy: a paradigm shift. J Natl Cancer Inst 105(4): 256-265.

Friedberg JW (2011) Relapsed/refractory diffuse large B-cell lymphoma. Hematology Am Soc Hematol Educ Program 2011: 498-505.

Garnett CT, Palena C, Chakraborty M, Tsang KY, Schlom J, Hodge JW (2004) Sublethal irradiation of human tumor cells modulates phenotype resulting in enhanced killing by cytotoxic T lymphocytes. Cancer Res 64(21): 7985-7994.

Gavet O, Pines J (2010) Progressive activation of CyclinB1-Cdk1 coordinates entry to mitosis. Dev Cell 18(4): 533-543.

Gelbard A, Garnett CT, Abrams SI, Patel V, Gutkind JS, Palena C, Tsang KY, Schlom J, Hodge JW (2006) Combination chemotherapy and radiation of human squamous cell carcinoma of the head and neck augments CTL-mediated lysis. Clin Cancer Res 12(6): 1897-1905.

Gloire G, Legrand-Poels S, Piette J (2006) NF-kappaB activation by reactive oxygen species: fifteen years later. Biochem Pharmacol 72(11): 1493-1505.

Hafer K, Iwamoto KS, Schiestl RH (2008) Refinement of the dichlorofluorescein assay for flow cytometric measurement of reactive oxygen species in irradiated and bystander cell populations. Radiat Res 169(4): 460-468.

Ithimakin S, Day KC, Malik F, Zen Q, Dawsey SJ, Bersano-Begey TF, Quraishi AA, Ignatoski KW, Daignault S, Davis A, Hall CL, Palanisamy N, Heath AN, Tawakkol N, Luther TK, Clouthier SG, Chadwick WA, Day ML, Kleer CG, Thomas DG, Hayes DF, Korkaya H, Wicha MS (2013) HER2 drives luminal breast cancer stem cells in the absence of HER2 amplification: implications for efficacy of adjuvant trastuzumab. Cancer Res 73(5): 1635-1646.

Junttila TT, Akita RW, Parsons K, Fields C, Lewis Phillips GD, Friedman LS, Sampath D, Sliwkowski MX (2009) Ligand-independent HER2/HER3/ PI3K complex is disrupted by trastuzumab and is effectively inhibited by the PI3K inhibitor GDC-0941. Cancer Cell 15(5): 429-440.

Kitazono M, Robey R, Zhan Z, Sarlis NJ, Skarulis MC, Aikou T, Bates S, Fojo $\mathrm{T}$ (2001) Low concentrations of the histone deacetylase inhibitor, depsipeptide (FR901228), increase expression of the $\mathrm{Na}(+) / \mathrm{I}(-)$ symporter and iodine accumulation in poorly differentiated thyroid carcinoma cells. J Clin Endocrinol Metab 86(7): 3430-3435.

Kute T, Lack CM, Willingham M, Bishwokama B, Williams H, Barrett K, Mitchell T, Vaughn JP (2004) Development of Herceptin resistance in breast cancer cells. Cytometry A 57(2): 86-93. 
Kwilas AR, Donahue RN, Bernstein MB, Hodge JW (2012) In the field: exploiting the untapped potential of immunogenic modulation by radiation in combination with immunotherapy for the treatment of cancer. Front Oncol 2: 104.

Lagadec C, Vlashi E, Della Donna L, Meng Y, Dekmezian C, Kim K, Pajonk F (2010) Survival and self-renewing capacity of breast cancer initiating cells during fractionated radiation treatment. Breast Cancer Res 12(1): R13.

Markovic A, Chung CH (2012) Current role of EGF receptor monoclonal antibodies and tyrosine kinase inhibitors in the management of head and neck squamous cell carcinoma. Exp Rev Anticancer Ther 12(9): 1149-1159.

Maruyama T, Mimura K, Izawa S, Inoue A, Shiba S, Watanabe M, Kawaguchi Y, Inoue M, Nogata H, Inoue S, Fujii H, Kono K (2011) Lapatinib enhances herceptin-mediated antibody-dependent cellular cytotoxicity by upregulation of cell surface HER2 expression. Anticancer Res 31(9): 2999-3005.

Mohd Sharial MS, Crown J, Hennessy BT (2012) Overcoming resistance and restoring sensitivity to HER2-targeted therapies in breast cancer. Ann Oncol 23(12): 3007-3016.

Pajonk F, Vlashi E, McBride WH (2010) Radiation resistance of cancer stem cells: the 4 R's of radiobiology revisited. Stem Cells 28(4): 639-648.

Phillips TM, McBride WH, Pajonk F (2006) The response of CD24(-/low)/ CD44 + breast cancer-initiating cells to radiation. J Natl Cancer Inst 98(24): 1777-1785.

Rajagopalan S, Willert E, Wirth R, Null W, Liu JS, Higgins JP (2013) Her2-targeted engineered toxin body demonstrates selective binding and cell kill of Her2-overexpressing breast cancer. AACR Annual Meeting: Washington DC, USA, abstract 868.

Reichert JM (2013) Antibodies to watch in 2013: Mid-year update. mAbs 5: 4.

Reits EA, Hodge JW, Herberts CA, Groothuis TA, Chakraborty M, Wansley EK, Camphausen K, Luiten RM, de Ru AH, Neijssen J, Griekspoor A, Mesman E, Verreck FA, Spits H, Schlom J, van Veelen P, Neefjes JJ (2006) Radiation modulates the peptide repertoire, enhances MHC class I expression, and induces successful antitumor immunotherapy. J Exp Med 203(5): 1259-1271.

Schneider CA, Rasband WS, Eliceiri KW (2012) NIH Image to ImageJ: 25 years of image analysis. Nat Methods 9(7): 671-675.
Scott AM, Allison JP, Wolchok JD (2012) Monoclonal antibodies in cancer therapy. Cancer Immunity 12: 14.

Shimizu R, Kikuchi J, Wada T, Ozawa K, Kano Y, Furukawa Y (2010) HDAC inhibitors augment cytotoxic activity of rituximab by upregulating CD20 expression on lymphoma cells. Leukemia 24(10): $1760-1768$.

Stagg J, Allard B (2013) Immunotherapeutic approaches in triple-negative breast cancer: latest research and clinical prospects. Ther Adv Med Oncol 5(3): 169-181.

Tagliabue E, Campiglio M, Pupa SM, Menard S, Balsari A (2012) Activity and resistance of trastuzumab according to different clinical settings. Cancer Treat Rev 38(3): 212-217.

Tejani MA, Cohen RB, Mehra R (2010) The contribution of cetuximab in the treatment of recurrent and/or metastatic head and neck cancer. Biol Targets Ther 4: 173-185.

Tsang WP, Chau SP, Kong SK, Fung KP, Kwok TT (2003) Reactive oxygen species mediate doxorubicin induced p53-independent apoptosis. Life Sci 73(16): 2047-2058.

Voutsas IF, Mahaira LG, Fotopoulou K, Kapranos N, Reclos JG, Gritzapis AD, Papamichail M, Perez SA, Baxevanis CN (2013) Gamma-irradiation induces HER-2/neu overexpression in breast cancer cell lines and sensitivity to treatment with trastuzumab. Int J Radiat Biol 89(5): 319-325.

Weiner LM, Dhodapkar MV, Ferrone S (2009) Monoclonal antibodies for cancer immunotherapy. Lancet 373(9668): 1033-1040.

Wirth R, Rajagopalan S, Willert E, Null W, Erdman J, Liu JS, Higgins JP (2013) Engineered toxin body demonstrating CD20-specific binding and cell kill in B-cell non-Hodgekin's lymphoma cells. AACR Annual Meeting: Washington DC, USA, abstract 5477.

This work is published under the standard license to publish agreement. After 12 months the work will become freely available and the license terms will switch to a Creative Commons AttributionNonCommercial-Share Alike 3.0 Unported License. 